\title{
Admission and Discharge Following Ambulance Transport to the Emergency Department
}

\author{
Aikaterini SAKAGIANNI ${ }^{\mathrm{a}, \dagger}$, Georgios FERETZAKIS ${ }^{\mathrm{b}, \mathrm{c}, \mathrm{d}, 1, \dagger}$, Georgios KARLIS $^{\mathrm{e}}$, \\ Evangelos LOUPELIS ${ }^{\mathrm{d}}$, Lazaros TZELVES ${ }^{\mathrm{f}}$, Rea CHATZIKYRIAKOU ${ }^{\mathrm{g}}$, \\ Nikolaos TRAKAS ${ }^{\mathrm{h}}$, Eugenia KARAKOU ${ }^{\mathrm{h}}$, Stavroula PETROPOULOU ${ }^{\mathrm{d}}$, \\ Aikaterini TIKA ${ }^{i}$, Zoi RAKOPOULOU ${ }^{i}$, Ilias DALAINAS ${ }^{i}$ and Vasileios KALDIS ${ }^{j}$ \\ ${ }^{a}$ Intensive Care Unit, Sismanogleio General Hospital, Marousi, Greece \\ ${ }^{\mathrm{b}}$ School of Science and Technology, Hellenic Open University, Patras, Greece \\ c Sismanogleio General Hospital, Department of Quality Control, Research and \\ Continuing Education, Marousi, Greece \\ d Sismanogleio General Hospital, IT department, Marousi, Greece \\ e Thoracic Diseases General Hospital Sotiria \\ ${ }^{\mathrm{f}}$ Second Department of Urology, National and Kapodistrian University of Athens, \\ Sismanogleio General Hospital, Athens, Greece \\ ${ }^{\mathrm{g}}$ Sismanogleio General Hospital, Hematology Laboratory, Marousi, Greece

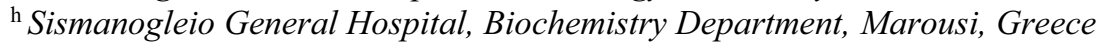 \\ i Sismanogleio General Hospital, Administration, Marousi, Greece

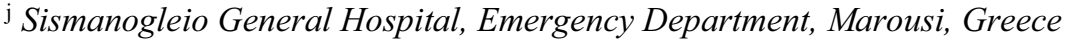

$\dagger$ Equal contribution (1st authors)

\begin{abstract}
Emergency ambulance use is deemed necessary for the transport of acutely ill patients to hospital emergency departments (ED). However, some patients are discharged as they present low acuity or chronic problems and should receive primary healthcare services, while the most severely ill are admitted. In the present study, we examined the descriptive epidemiology of ambulance transports for emergencies in the ED by utilizing the data of the information systems of a public tertiary general hospital in Greece. More than half of the patients transferred to the ED by an ambulance were finally admitted to the hospital $(52.25 \%)$, whereas only one-third $(33.74 \%)$ of those transferred by other means. A statistically significant association was detected between ambulance use and hospital admission. Age was also statistically significantly higher in the ambulance group. Higher mean values of creatinine, CRP, LDH, urea, white-blood-cell count, and neutrophils were detected in the ambulance group, in contrast to hemoglobin and lymphocyte count which were higher in the non-ambulance group.
\end{abstract}

Keywords: emergency medical services, ambulances, hospital information systems

\footnotetext{
${ }^{1}$ Corresponding Author, Georgios FERETZAKIS, PhD, E-mail address: georgios.feretzakis@ac.eap.gr
} 


\section{Introduction and Background}

Emergency ambulance use is usually reserved for transporting acutely ill patients to hospital emergency departments (ED). However, a substantial proportion of ambulance users are discharged as they present low acuity or chronic problems and could benefit from primary healthcare services, while the most severely ill are admitted to hospitals [1-3]. A significant increase in ambulance demand has been observed worldwide, reaching $29.2 \%$, in a recent 8 -year observational study in Melbourne, Australia [4]. Several articles in the literature have tried to elucidate the phenomenon of increased use of emergent care services by patients with non-acute situations [5-7]. Factors as older age, low socioeconomic status, and homelessness have been suggested [6,7]. Similarly, non-emergent ambulance calls by patients with minor illnesses may have severe implications regarding medical resources adequacy for those who really need them [8]. Moreover, increased ambulance transport of patients with primary care medical problems leads to the overcrowding of hospital emergency departments and potentially to the provision of lower quality healthcare services [5]. The aim of this study was to compare the characteristics of patients visiting the ED in relation to the use of ambulance transport and the ED outcome (admission or discharge).

\section{Materials and Methods}

This research is an observational retrospective statistical analysis conducted in the ED of a public tertiary care hospital in Greece that has been approved by the Institutional Review Board of Sismanogleio General Hospital (Ref. No 15177/2020, 5969/2021). Between 14 March and 4 May 2019, 2903 ED visits were recorded (missing values of the Ambulance variable were excluded from the statistical analysis). We investigated biochemical markers and coagulation tests that are routinely checked in patients visiting the ED, in relation to the use of ambulance transport and the ED outcome (admission or discharge). Raw data were retrieved from a standard Laboratory Information System (LIS) and a hospital information system (HIS). The data were analyzed by using IBM SPSS Statistics version 27.0 [9]. The data set includes the following variables: ambulance use (Ambulance), serum levels of Urea (UREA), Creatinine (CREA), Lactate Dehydrogenase (LDH), Creatine Kinase (CPK), C-Reactive Protein (CRP), Complete Blood Count with differential, including white blood cells (WBC), neutrophil count (NEUT\%), lymphocyte count (LYM\%), hemoglobin (HGB), and platelets (PLT), Activated Partial Thromboplastin Time (aPTT), D-Dimer, International Normalized Ratio (INR), age, gender, and triage disposition to ED unit.

The descriptive statistics of the data for the variables Age, Gender, Ambulance use, and Admission are presented in the following table (Table 1).

Table 1. Summary of descriptive statistics for variables Age, Gender, Ambulance use, and Admission

\begin{tabular}{|l|c||c|c||c|c||c|c|}
\hline \multicolumn{2}{|c||}{ Age } & \multicolumn{2}{c||}{ Gender } & \multicolumn{2}{c|}{ Ambulance use } & \multicolumn{2}{c|}{ Admission } \\
\hline Mean & 61.17 & Male & 1453 & Yes & 511 & Yes & 1074 \\
\hline Standard deviation & 20.82 & Female & 1450 & No & 2392 & No & 1829 \\
\hline Range/IQR & $86 / 33$ & Total & 2903 & Total & 2903 & Total & 2903 \\
\hline
\end{tabular}


The patient factors were compared between the ambulance and non-ambulance groups using Pearson's Chi-squared test for categorical variables and independent sample t-test for continuous data, both with a 0.05 level of significance.

\section{Results}

A total of 2903 patients visited the ED from March 14th until May 4th, 2019, with a 1:1 ratio of males to females. The admission rate was equal to $37 \%$, while the rest $63 \%$ was discharged. Among the 2903 ED patients, only $17.6 \%(n=511)$ used an ambulance. Patients transported by an ambulance had a higher probability of being admitted to the hospital compared to those who did not use emergency medical services (OR 2.15, $95 \%$ CI $1.772-2.607)$. More than half $(52.25 \%)$ of the patients transported by an ambulance were admitted to the hospital. The rest of them were discharged as their condition was not deemed urgent. Among patients not transferred to the hospital by ambulance, $33.74 \%$ were admitted.

The majority of patients that visited ED (93\%) were referred to specific units. About $41 \%(n=1118)$ were referred to the internal medicine unit, $23.3 \%(n=630)$ to the pulmonology unit, and $15.7 \%(n=321)$ to the cardiology unit. Among those transferred to the ED by ambulance, $92.3 \%$ (472 out of 511) were referred to a specific ED unit. The respective percentages were $43.2 \%(n=204)$ with medical problems, $23 \%(n=109)$ with respiratory problems, and $21.8 \%(\mathrm{n}=103)$ with heart-related problems.

Chi-square analysis showed that the use of ambulance and hospital admission had a statistically significant association $\left(\mathrm{X}^{2}=61.911, \mathrm{p}<0.001\right)$. Phi and Cramer's V values were 0.146 , which indicates a weak association. On the other side, our statistical analysis showed no statistically significant association between gender and ambulance use $\left(\mathrm{X}^{2}=\right.$ $0.170, p=0.680>0.05)$. Independent samples t-tests were performed between the ambulance and non-ambulance groups and deduced that the means of the following continuous variables were statistically significantly higher in the ambulance group: CREA, CRP, LDH, UREA, WBC, and NEUT\%. On the contrary, the means of HGB and LYM\% were statistically significantly lower in the ambulance group. No statistically significant difference was observed between the two groups regarding the other continuous variables. A detailed summary of the independent samples t-tests is presented in the following table (Table 2). Age was also statistically significantly higher in the ambulance group, with a mean difference of 13.11 years, compared to patients not using an ambulance.

Table 2. Summary of the independent samples t-tests in laboratory testing data between the ambulance and non-ambulance groups

\begin{tabular}{|l||c|c|c|c|c|c|c|c|}
\hline & CREA & CRP & LDH & UREA & HGB & LYM\% & NEUT\% & WBC \\
\hline $\begin{array}{l}\text { Mean } \\
\text { Difference }\end{array}$ & -0.2718 & -16.625 & -45.630 & -13.768 & 0.875 & 5.229 & -6.111 & -1.114 \\
\hline t- value & -4.931 & -4.139 & -3.729 & -7.340 & 7.522 & 9.364 & -9.762 & -4.351 \\
\hline p-value & $<0.001$ & $<0.001$ & $<0.001$ & $<0.001$ & $<0.001$ & $<0.001$ & $<0.001$ & $<0.001$ \\
\hline
\end{tabular}




\section{Discussion}

The emergency medical service (EMS), worldwide, is ruled from several life-threatening level protocols of time response. This is a useful tool when the demand for services exceeds resource availability, and it is therefore vital that the appropriate transport request is made. In Greece, the public ambulance service covers the transportation of acutely ill patients to hospitals, as well as scheduled local and interregional urgent and non-urgent inter-hospital patient transfers. The present study examined the descriptive epidemiology of EMS ambulance transports in the ED by utilizing the HIS and LIS from a public tertiary general Greek hospital. Demographic inpatient data were merged with patients' laboratory data of the emergency department, elaborating information on whether the patient arrived by ambulance or not. Older patients and those with more deranged laboratory exams had a greater possibility to use an ambulance service as a lifethreatening medical emergency code. However, only half of the patients transported by an ambulance were admitted to the hospital, whereas only one-third of those were not transported by an ambulance. Nevertheless, a medical fast-track service is available in the triage room, permitting patients with minor medical issues to avoid unnecessary laboratory or imaging tests, therefore diminishing their discharge time. From the total number of patients visiting the ED, nearly two-thirds were discharged, meaning that they presented with mild problems, which could be initially managed at primary healthcare services. Although ambulance users are more frequently older, with more severe illness and greater need for admission, in approximately half of the cases, EMS ambulance call was actually unnecessary, and patients were discharged, possibly due to minor or chronic problems. Our results are in accordance with similar studies $[1,3,5]$. The optimal use of public, pro bono emergency ambulance services by the citizens is desirable as it could help alleviate ED crowding and improve the quality of emergency hospital services. An association between poor patient outcomes and ED overcrowding has been suggested [5]. The role of primary healthcare services should also be strengthened as it is fundamental in achieving better health care services in general.

\section{References}

[1] Yazaki H, Nishiura H. Ambulance Transport of Patients with Mild Conditions in Hokkaido, Japan. Int J Environ Res Public Health. 2020 Feb 2;17(3):919. doi: 10.3390/ijerph17030919.

[2] Ting JY, Chang AM. Path analysis modeling indicates free transport increases ambulance use for minor indications. Prehosp Emerg Care. 2006 Oct-Dec;10(4):476-81. doi: 10.1080/10903120600885209.

[3] Hegenberg K, et al. Differences between cases admitted to hospital and discharged from the emergency department after emergency medical services transport. BMJ Open. 2019 Sep 27;9(9):e030636.

[4] Morley C, Unwin M, Peterson GM, Stankovich J, Kinsman L. Emergency department crowding: A systematic review of causes, consequences and solutions. PLoS One. 2018 Aug 30;13(8):e0203316.

[5] Unwin M, Crisp E, Rigby S, Kinsman L. Investigating the referral of patients with non-urgent conditions to a regional Australian emergency department: a study protocol. BMC Health Serv Res. 2018 Aug 20;18(1):647. doi: 10.1186/s12913-018-3411-4.

[6] Andrew E, Nehme Z, Cameron P, Smith K. Drivers of Increasing Emergency Ambulance Demand. Prehosp Emerg Care. 2020 May-Jun;24(3):385. doi: 10.1080/10903127.2019.1635670.

[7] Coster JE, Turner JK, Bradbury D, Cantrell A. Why Do People Choose Emergency and Urgent Care Services? A Rapid Review Utilizing a Systematic Literature Search and Narrative Synthesis. Acad Emerg Med. 2017 Sep;24(9):1137-1149. doi: 10.1111/acem.13220.

[8] Norberg G, Wireklint Sundström B, Christensson L, Nyström M, Herlitz J. Swedish emergency medical services' identification of potential candidates for primary healthcare: Retrospective patient record study. Scand J Prim Health Care. 2015;33(4):311-7. doi: 10.3109/02813432.2015.1114347.

[9] IBM Corp. Released 2020. IBM SPSS Statistics for Windows, Version 27.0. Armonk, NY: IBM Corp 